\title{
Avaliação do raciocínio abstrato em estudantes do ensino médio ${ }^{1}$
}

\author{
Wagner Bandeira Andriola \\ Luanna Rodrigues Cavalcante
}

Universidade Federal do Ceará

\begin{abstract}
Resumo
A presente pesquisa objetivou avaliar a capacidade cognitiva denominada de raciocínio abstrato em estudantes do ensino médio. Tal construto refere-se à capacidade que o indivíduo possui para resolver problemas compostos por analogias em formato de símbolos abstratos. Utilizaram-se 513 estudantes do ensino médio de escolas públicas e particulares de Fortaleza (CE) submetendo-os ao Teste de Raciocínio Abstrato (RA), desenvolvido por W. B. Andriola, em 1996. Através da análise de variância (ANOVA), compararam-se as variáveis sexo, idade, tipo de escola e série escolar quanto ao desempenho no Teste RA.
\end{abstract}

Palavras-chave: raciocínio abstrato, avaliação psicológica, cognição
Key-words

\begin{abstract}
Evaluation of the abstract reasoning in students of the secondary level. This research aimed the evaluation of the cognitive capacity denominated Abstract Reasoning using students of the secondary level. It deals with our capacity to solve problems composed by abstract symbols. 513 students of the secondary level from public and private schools in the city of Fortaleza (CE, Northeast of Brazil) made up the sample, while the instrument used was the Abstract Reasoning Test developed by W. B. Andriola in 1996. By using analysis of variance (ANOVA) it was possible to compare the variables: sex, age, type of school and academic level to the performance on the Abstract Reasoning Test.
\end{abstract}


$\mathrm{P}$ ara se entender a origem dos testes e a importância de traba lhos que objetivam a avaliação das capacidades cognitivas, em especial a denominada de raciocínio abstrato, faz-se necessária uma breve recapitulação de alguns aspectos históricos da psicometria.

Das preocupações pertinentes à psicometria, duas, inicialmente independentes, podem ser destacadas: a prática e a teórica. A preocupação prática deu ênfase à avaliação dos aspectos psicopedagógicos e clínicos com o objetivo principal de detectar, através de provas psicológicas, o retardo mental e o potencial dos sujeitos com fins de predição na área acadêmica (Pasquali, 1997).

A preocupação teórica teve como objetivo principal o desenvolvimento da própria teoria psicométrica, sobretudo por psicólogos de orientação estatística. Por volta do ano de 1940 essa cisão tem seu fim, devido a influência dos psicólogos que utilizavam a análise fatorial (Pasquali, 1997).

O campo em que a psicometria se originou e mais se desenvolveu foi o dos testes psicológicos, que foram surgindo no final do século passado. Os problemas existentes na educação deram grande impulso a esse desenvolvimento. Os testes foram empregados na classificação das crianças de acordo com o aproveitamento escolar; na identificação de pólos na intelectualidade (retardados/dotados); no diagnóstico dos fracassos acadêmicos; no aconselhamento educacional e vocacional e na seleção de candidatos para escolas profissionais e outras escolas especiais (Anastasi, 1977).

Segundo Anastasi (1977), a história da avaliação das capacidades cognitivas está ligada às necessidades sociais, de explicação do comportamento humano, e educacionais, que objetivam a melhoria do rendimento escolar dos alunos. Nesse sentido, Vianna (1982) destaca que: 1) a medida do desempenho escolar é fundamental para uma educação eficiente; 2) os instrumentos de medida facilitam a observação realizada pelo professor acerca do desempenho dos alunos; e 3) todos os objetivos educacionais podem ser mensurados.

Assim, a construção e a utilização dos instrumentos de medida devem ser incentivadas, com o cuidado de levar em consideração a validação dos mesmos e principalmente a formação dos psicólogos 
que irão trabalhar nessa área. Klausnitzer (1985) ressalta ainda que "de todos os chamados testes psicológicos atualmente existentes no país, somente um número pequeno corresponde às mínimas exigências científicas" (pp. 15-16).

Atualmente, as investigações tomam variáveis cognitivas mais processuais e não exclusivamente as resultantes da aplicação dos testes de inteligência ou de Q. I., que vislumbravam aspectos puramente quantitativos. Há uma maior preocupação com a interação de diversas variáveis (sexo, idade, classe social, comunidade rural ou urbana, etnia) antes consideradas isoladamente (Almeida, 1988a).

\section{Definição e caracterização do raciocínio}

O conceito "raciocínio", segundo Almeida (1988b), é associado a outros conceitos, como inteligência, resolução de problemas e compreensão/pensamento. É abordado por diversas perspectivas teóricas tais como a desenvolvimentalista, a cognitivista e a diferencial (Almeida, 1988b).

Na tentativa de delimitar o construto raciocínio, far-se-á uma breve diferenciação de conceitos, seguida por uma descrição do mesmo, tal como é visto pela perspectiva cognitivista.

Segundo Almeida (1988b), se o conceito de inteligência abrange todos os processos cognitivos que vão desde a recepção da informação até a elaboração da resposta, passando pelos processos de codificação, memorização, aprendizagem, evocação e relacionamento da informação, o raciocínio é responsável pelos componentes de tratamento e relacionamento da informação.

Quanto à diferenciação dos conceitos de "raciocínio" e de "resolução de problemas", Almeida (1988b) destaca o caráter mais abrangente do segundo. Enfatiza que o raciocínio é um dos elementos mais importantes da resolução de problemas, principalmente quando as tarefas a serem resolvidas exigirem descobertas de relações, comparação de elementos e a escolha de uma entre várias alternativas propostas como resposta.

Segundo o mesmo, o termo "compreensão/pensamento" está ligado ao problema e aos conhecimentos, enquanto que o "raciocínio" está relacionado a apreensão e aplicação de relações. 
Diante dessas diferenciações, pode-se concluir que os conceitos de "inteligência", "resolução de problemas" e "compreensão/pensamento" são mais abrangentes e estão relacionados à resolução de tarefas, de uma maneira geral, o que não exclui, segundo Almeida (1988b), a possibilidade de algumas dessas tarefas exigirem o raciocínio.

Dessa forma, o raciocínio pode ser encarado como uma capacidade cognitiva exigida na resolução de problemas simples e complexos, tanto de ordem intelectual como de situações quotidianas. Vale ressaltar dois importantes fatores que influenciam na resolução de problemas: a natureza da tarefa e o tipo de conhecimento trazido pelo indivíduo para a resolução dos mesmos (Chi \& Glaser, 1992).

Para Almeida (1988b), na perspectiva cognitivista a análise centrase nas tarefas e nos processos cognitivos internos usados para a resolução dos problemas, enquanto que o raciocínio seria caracterizado por:

1. identificar os elementos de uma tarefa ou problema;

2. retirar conclusões lógicas da informação fornecida e processada;

3. compreender a sua formulação;

4. conceber formas alternativas de resolução;

5. avaliar as diferentes alternativas de resolução;

6. avaliar a adequação da resposta dada ao problema, considerando a especificidade da situação e suas consequiências.

Pode-se dizer com isso, que o raciocínio é um mecanismo cognitivo utilizado para solucionar problemas em diferentes formas de conteúdo (numérico, verbal, espacial, abstrato e mecânico). Tratando-se especificamente do raciocínio abstrato, pode-se dizer que se caracteriza pela capacidade de resolver problemas compostos por símbolos abstratos.

Abordando a importância da utilização do raciocínio abstrato por estudantes do ensino médio, pode-se destacar as disciplinas de matemática e física, que são obrigatórias nos currículos escolares e exigem um elevado grau de capacidade para perceber e aplicar relações entre fenômenos e símbolos abstratos.

\section{Estudos realizados na área}

$\mathrm{Na}$ área de avaliação do raciocínio pode-se destacar o estudo realizado por Andriola (1995a), cujo objetivo foi avaliar a capacida- 
de de raciocinar com símbolos numéricos em estudantes do ensino médio. A amostra utilizada foi composta por 545 estudantes secundaristas e o instrumento foi o Teste de Raciocínio Numérico (RN) desenvolvido por Andriola (1996b). Trata-se de um teste de medida psicológica de apuração objetiva, composto por 25 seqüências numéricas e com tempo limite de 10 minutos para a sua resolução.

Para a comparação do desempenho dos respondentes no Teste $\mathrm{RN}$ foi utilizada a análise de variância, cujos resultados indicaram que:

1. considerando-se o sexo, detectou-se uma diferença significativa $\left[F_{(1,524)}=30,30 ; p<0,01\right]$. Assim, os homens obtiveram desempenho superior às mulheres;

2. considerando-se a série escolar, detectaram-se diferenças significativas $\left[\mathrm{F}_{(2,530)}=20,70 ; \mathrm{p}<0,01\right]$. Comparativamente, os alunos da $1^{a}$ série obtiveram desempenhos superiores aos da $2^{\mathrm{a}}$ e $3^{\mathrm{a}}$;

3. considerando-se a idade ${ }^{2}$, detectaram-se diferenças significativas $\left[F_{(2,524)}=39,11 ; p<0,01\right]$. Os sujeitos das faixas 1 e 2 obtiveram desempenhos superiores aos da 3 .

Diante desse resultados, a explicação provável para a diferença a favor dos estudantes do sexo masculino pode se dever a uma evolução mais rápida quanto à aptidão numérica ou um maior sucesso escolar na matemática e nas ciências. Quanto às diferenças entre as faixas etárias e as séries escolares, no sentido de que os estudantes mais novos obtiveram desempenhos mais elevados que os mais velhos, pode ser explicada pelo fato dos mais velhos, provavelmente, terem um número maior de repetências escolares e menor nível de envolvimento na resolução das tarefas.

Em outro estudo, com o objetivo de avaliar a capacidade de raciocinar utilizando símbolos verbais, Andriola (1997) utilizou uma amostra composta por 658 estudantes secundaristas submetidos ao Teste de Raciocínio Verbal (RV), composto por 40 analogias verbais e com tempo limite para a sua resolução de 10 minutos.

Para a comparação do desempenho dos respondentes no Teste RV foi utilizada a análise de variância, cujos resultados indicaram que: 
1. considerando-se o sexo, não detectou-se diferença significativa $\left[\mathrm{F}_{(1,}\right.$ $\left.{ }_{656)}=0,005 ; \mathrm{p}<0,01\right]$. Comparativamente, não houve diferenças entre os desempenhos de homens e mulheres no Teste RV;

2. considerando-se a série escolar, não detectaram-se diferenças significativas $\left[\mathrm{F}_{(2,655)}=1,505, \mathrm{p}<0,01\right]$. Comparativamente, os alunos da $1^{\mathrm{a}}, 2^{\mathrm{a}}$ e $3^{\mathrm{a}}$ séries obtiveram o mesmo desempenho;

3. considerando-se a idade ${ }^{3}$, não detectaram-se diferenças significativas $\left[F_{(2,655)}=0,856\right]$. Os sujeitos das faixas 1,2 e 3 obtiveram o mesmo desempenho.

Pode-se concluir com isso, que a amostra utilizada era extremamente homogênea quanto à capacidade avaliada, visto que nenhuma das diferenças encontradas na comparação do desempenho com as variáveis sexo, série escolar e faixa etária foram significativas.

Almeida (1988b) realizou estudo objetivando estabelecer a relação entre o resultado nas provas que constituíam a Bateria de Provas de Raciocínio Diferencial (BPRD), destinada aos alunos do ensino secundário de escolas portuguesas, e o sucesso escolar dos mesmos. Os resultados indicaram que melhores desempenhos nas provas correspondiam a classificações escolares superiores, a menor taxa de insucesso escolar no final do ano letivo em que as provas foram aplicadas e a um menor número de reprovações escolares em anos anteriores.

Diante do exposto, a presente pesquisa objetivou avaliar a capacidade de raciocinar com símbolos abstratos em estudantes do ensino médio da cidade de Fortaleza. Os escores obtidos no Teste RA foram comparados em termos de sexo, idade, tipo de escola e série escolar.

\section{A escolha do instrumento}

Segundo Andriola (1996a), atualmente, a área de avaliação psicológica está tendo uma visão mais crítica quanto ao uso de testes psicológicos. Estudos estão sendo desenvolvidos visando adaptações, construções e informatizações de instrumentos psicológicos à realidade brasileira. Entre os anos 30 e 60, a construção de testes no Brasil teve seu auge. Contudo, alguns testes não tiveram seus parâmetros psicométricos determinados, pois seus criadores relegaram tal determinação a segundo plano (Andriola, 1995b). 
Deve-se considerar, ainda, a existência de vários erros sobre os resultados dos estudos que foram realizados nessa época, com relação à determinação dos parâmetros psicométricos, pois o uso das técnicas estatísticas era bastante precário. A utilização do computador para a escolha amostral e para as análises estatísticas ainda não era realizada (Andriola, 1995b).

Ressalte-se que na formação do psicólogo há, segundo Japur (apud Andriola, 1996a), uma ênfase demasiada na formação clínica do estudante de psicologia, sendo relegada a plano secundário a necessidade de preparação de profissionais qualificados na área de avaliação e medida psicológica.

Sendo assim, a determinação dos parâmetros métricos dos instrumentos de medida psicológica reveste-se de extrema importância, objetivando-se evitar ao máximo erros e equívocos que são cometidos ao se usar um material padronizado em outro país.

O instrumento escolhido para a presente pesquisa foi o Teste de Raciocínio Abstrato (RA), desenvolvido por W. B. Andriola, em 1996, por ter seus parâmetros métricos determinados para a nossa realidade.

A validade fatorial realizada, inicialmente, através do método de extração dos componentes principais indicou a presença de quatro fatores, explicando juntos $43,2 \%$ da variância total, cujos valores próprios eram superiores a um. Nova análise fatorial foi feita, desta vez para extrair um único fator, que explicou $29,2 \%$ da variância total, com valor próprio de 8,5 .

A precisão do instrumento foi determinada através do Alpha de Cronbach, que resultou no valor 0,90 , ou seja, o Teste RA possui elevada precisão para avaliar o raciocínio abstrato de estudantes do ensino médio.

\section{Método}

\section{Amostra}

Utilizou-se uma amostra de 513 estudantes oriundos de escolas públicas e particulares, da cidade de Fortaleza, com idade média de 17,15 anos $(\mathrm{dp}=1,9)$, sendo a maior parte do sexo feminino $(57,8 \%)$ e da $1^{\text {a }}$ série do ensino médio $(47,8 \%)$. 
Um dos problemas presente na maioria das pesquisas em Psicologia é a falta de representatividade da amostra. Revela-se, assim, a necessidade de se aplicar modelos matemáticos que permitam determinar o tamanho de uma amostra representativa da população. $\mathrm{O}$ cuidado com este aspecto proporciona ao pesquisador elevado grau de confiabilidade quanto às generalizações dos resultados de seu trabalho.

De acordo com Conboy (1995), uma das maneiras de comprovar a representatividade amostral é através da determinação do erro cometido. Segundo Trompieri Filho, Nóbrega e Andriola (1995), para efetivar tal cálculo, algumas suposições têm que ser feitas quanto a variável medida (raciocínio abstrato):

a. pode ser dicotomizada;

b. tem distribuição normal na população (Média=0, $\mathrm{DP}=1$ );

c. a variância máxima assume o valor 0,25 .

Adotando-se o intervalo de confiança de $95 \%$, isto é, a $=0,05$ e aplicando-se estes valores na fórmula ${ }^{4}$ obtém-se um erro de $4,3 \%$, ou seja, há uma aceitável garantia de representatividade da amostra e, portanto, de que os resultados podem ser generalizados para a população (Conboy, 1995).

\section{Instrumento}

O instrumento utilizado foi o Teste de Raciocínio Abstrato (RA), construído por W. B. Andriola, em 1996. Trata-se de um instrumento de apuração objetiva, composto por 30 questões em formato de analogias e com tempo limite de 15 minutos para a sua resolução ${ }^{5}$.

\section{Procedimento}

O instrumento foi aplicado junto aos estudantes em horários preestabelecidos. As instruções necessárias foram dadas, de forma que os estudantes respondessem ao instrumento.

\section{Resultados}

Os dados foram organizados em um banco de dados e analisados estatisticamente através do pacote SPSS for Windows (7.5). 
A análise descritiva do desempenho dos 513 respondentes revelou que a média de acertos no Teste RA foi de 20,85 (dp=6,29), com amplitude variando de 2 a 29. Outras análises foram realizadas objetivando comparações quanto ao sexo, série escolar, tipo de escola e idade dos respondentes. A Tabela 1 apresenta o desempenho dos estudantes levando-se em consideração a variável sexo.

Tabela 1

Estatística descritiva do desempenho de homens e mulheres no Teste RA

\begin{tabular}{lccc}
\hline & Média & Desvio-padrão & Amplitude \\
\hline Homens $(\mathrm{n}=231)$ & 20,54 & 6,36 & $2-29$ \\
Mulheres $(\mathrm{n}=281)$ & 21,08 & 6,23 & $2-29$ \\
\hline
\end{tabular}

Os dados da Tabela 1 revelam que as mulheres obtiveram desempenho médio superior aos homens, com variabilidade menor. Utilizou-se o teste da análise de variância (ANOVA - One Way) para verificar se as diferenças observadas eram estatisticamente significativas. $\mathrm{O}$ resultado obtido $\left[\mathrm{F}_{(1,511)}=0,94 ;\right.$ n.s. $]$ indicou não haver diferença significativa entre os sexos.

Tabela 2

Estatística descritiva do desempenho dos respondentes no Teste RA segundo a série escolar

\begin{tabular}{lccc}
\hline & Média & Desvio-padrão & Amplitude \\
\hline $1^{2}$ série $(n=245)$ & 20,54 & 6,48 & $4-29$ \\
$2^{\text {a }}$ série $(n=92)$ & 20,45 & 6,29 & $2-29$ \\
$3^{\text {a }}$ série $(n+175)$ & 21,45 & 6,01 & $4-29$ \\
\hline
\end{tabular}

A Tabela 2 apresenta o desempenho dos estudantes levando-se em consideração a variável série escolar.

De acordo com os dados da Tabela 2, observa-se que os alunos da $3^{\mathrm{a}}$ série obtiveram desempenho médio superior às demais séries, com variabilidade menor. Utilizou-se o teste da análise de variância para verificar se as diferenças observadas eram estatisticamente sig- 
nificativas. O resultado obtido $\left[\mathrm{F}_{(2,511)}=1,32\right.$; n.s. $]$ indicou não haver diferenças significativas entre as séries.

Tabela 3

Estatística descritiva do desempenho dos respondentes no Teste RA segundo o tipo de escola

\begin{tabular}{lccc}
\hline & Média & Desvio-padrão & Amplitude \\
\hline Pública $(\mathrm{n}=256)$ & 19,08 & 6,48 & $2-28$ \\
Particular $(\mathrm{n}=256)$ & 22,59 & 5,57 & $2-29$ \\
\hline
\end{tabular}

A Tabela 3 apresenta o desempenho dos estudantes levando-se em consideração a variável tipo de escola.

De acordo com os dados constantes na Tabela 3, observa-se que os alunos das escolas particulares obtiveram desempenho médio superior aos da escola pública, com menor variabilidade. O teste da análise de variância indica que as diferenças observadas eram estatisticamente significativas $\left[\mathrm{F}_{(1,511)}=43,22 ; \mathrm{p}<0,05\right]$.

Para a comparação das idades foram organizadas cinco faixas etárias: 1) 14-15anos; 2) 16 anos; 3) 17 anos; 4) 18 anos; e 5) mais de 19 anos. A Tabela 4 apresenta o desempenho dos estudantes levando-se em consideração a variável idade.

Tabela 4

Média e desvio-padrão dos resultados no Teste $R A$ segundo a idade $(n=513)$

\begin{tabular}{lccc}
\hline & Média & Desvio-padrão & Amplitude \\
\hline Idade $1(n=84)$ & 22,65 & 5,73 & $5-29$ \\
Idade 2 $(n=114)$ & 21,42 & 6,18 & $4-29$ \\
Idade 3 $(n=127)$ & 21,58 & 6,15 & $2-28$ \\
Idade 4 $(n=94)$ & 19,83 & 6,67 & $2-28$ \\
Idade 5 $(n=92)$ & 18,66 & 5,72 & $5-29$ \\
\hline
\end{tabular}

Observou-se que os alunos mais novos (Idade 1) obtiveram desempenho médio superior às outras quatro faixas etárias. A menor variabilidade foi observada na Idade 5. Utilizou-se o teste da análise de variância para verificar se as diferenças observadas eram estatis- 
ticamente significativas. $\mathrm{O}$ resultado obtido $\left[\mathrm{F}_{(4,510)}=6,12 ; \mathrm{p}<0,05\right]$ indicou haver diferenças significativas entre as faixas etárias. Houve diferenças entre as Idades 1 e 3; 1 e 4; 1 e 5; e, 2 e 4, ou seja, os mais novos tem desempenho superior aos mais velhos.

\section{Discussão}

Sendo o raciocínio a capacidade cognitiva exigida na resolução de problemas simples e complexos, tanto de ordem intelectual como de situações quotidianas, a relevância da sua avaliação centra-se na possibilidade de explicação de comportamentos educacionais que objetivem a melhoria do rendimento escolar dos alunos.

Especificamente em relação à avaliação do raciocínio abstrato, pode-se destacar a importância que assume para os que estudam, principalmente, matemática e física, pelo fato de essas disciplinas exigirem um elevado grau de capacidade para perceber e aplicar relações entre fenômenos e símbolos abstratos. Medidas educacionais podem ser adotadas a partir de uma avaliação como a realizada.

Destaque-se a preocupação que se teve em utilizar um teste com características métricas determinadas para a nossa realidade, na tentativa de minimizar ao máximo erros inerentes ao processo de avaliação psicológica.

Sobre os resultados obtidos nessa pesquisa, observou-se a existência de diferenças entre as faixas etárias (os estudantes mais novos tiveram um melhor desempenho) e entre o tipo de escola (os estudantes das escolas particulares saíram-se melhor do que os das escolas públicas). O declínio no desempenho cognitivo de estudantes mais velhos corrobora os resultados de Almeida (1988a). Segundo Andriola (1995a), esse fato pode ser explicado pelo maior número de repetências escolares e a possibilidade de um menor nível de envolvimento com a resolução das tarefas.

Para os alunos de escolas particulares, os estímulos, caracterizados pelos tipos de itens presentes no Teste RA, possibilitaram melhores desempenhos, provavelmente pela similaridade com os tipos de atividades que eles já desenvolvem. Não se pode deixar de levar em consideração as diferenças quanto ao nível socioeconômico. 
Almeida (1988a) identificou desempenhos superiores por parte de alunos das classes sociais mais elevadas, principalmente nas tarefas de conteúdo verbal e abstrato.

Quanto ao resultado obtido na comparação entre os sexos, observou-se diferença não-significativa, o que corrobora os resultados obtidos por Almeida (1988b).

Assim, os resultados aqui encontrados possibilitam a execução de outros estudos objetivando identificar, efetivamente, as variáveis responsáveis pelas diferenças detectadas, sobretudo, quanto ao tipo de escola e idade.

\section{Referências}

Almeida, L. S. (1988a). Diferenças de sexo e de classe social em testes de raciocínio: estudo junto de alunos do ensino secundário. Revista Portuguesa de Pedagogia, XXII, 111-139.

Almeida, L. S. (1988b). O raciocínio diferencial dos jovens. Porto: Instituto Nacional de Investigação Científica.

Almeida, L. S. (1988c). Teorias da inteligência. Porto: Edições Jornal de Psicologia.

Anastasi, A. (1977). Testes Psicológicos (2 $2^{\mathrm{a}}$ ed., D. M. Leite, Trad.). São Paulo: EPU.

Andriola, W. B. (1995a). Avaliação do raciocínio numérico em estudantes do 2 grau. Educação em Debate, 17/18 (29-32), 95-99.

Andriola, W. B. (1995b). Problemas e perspectivas quanto ao uso dos testes psicológicos no Brasil. Psique, 5(6), 46-57.

Andriola, W. B. (1996a). Avaliação psicológica no Brasil: considerações a respeito da formação dos psicólogos e dos instrumentos utilizados. Psique, 6(8), 98-109.

Andriola, W. B. (1996b). A construção de um Teste de Raciocínio Numérico (RN). Avaliação Psicológica: Formas e Contextos, 4, 149-156.

Andriola, W. B. (1997). Avaliação do raciocínio verbal em estudantes do $2^{\circ}$ grau. Estudos de Psicologia, 2(2), 277-285.

Chi, M. T. H., \& Glaser, R. (1992). A capacidade para a resolução de problemas. In R. J. Sternberg (Org.), As capacidades intelectuais humanas (pp. 250-275). Porto Alegre: Artes Médicas.

Conboy, J. (1995). A estimativa da magnitude do $N$ de uma amostra. Aveiro: CIDINE.

Klausnitzer, J. E. (1985). Escola de inteligência. Rio de Janeiro: Tecnoprint. 
Pasquali, L. (1997). Psicometria: teoria e aplicações. Brasília: Editora Universidade de Brasília.

Trompiere Filho, N., Nóbrega, A. M. V., \& Andriola, W. B. (1995). Análise métrica da ficha de avaliação docente utilizada pela Universidade Federal do Ceará. Educação em Debate, 17/18, 29-32. Vianna, H. M. (1982). Testes em Educação. São Paulo: Ibrasa.

Wagner Bandeira Andriola, especialista em Psicometria e mestre em Psicologia Social e do Trabalho pela Universidade de Brasília, é professor do Departamento de Fundamentos da Educação, Mestrado em Avaliação Educacional, da Faculdade de Educa-
1 Pesquisa realizada no Laboratório de Pesquisas em Avaliação e Medida Psico-Educacional (LABPAM)

2 As idades foram organizadas em três faixas etárias: (a) entre 13 e 16 anos; (b) entre 17 e 18 anos e (c) mais de 19 anos.

3 As idades foram organizadas em três faixas etárias: (a) entre 13 e 15 anos; (b) entre 16 e 18 anos e (c) mais de 19 anos.

4 Notação: $\mathrm{e}=$ erro de estimativa; $\mathrm{z}=$ valor absoluto dos limites da área na distribuição normal reduzida correspondente à confiança de 95\%; $\mathrm{n}=$ tamanho da amostra utilizada no estudo; $\mathrm{s}=$ valor máximo do desvio padrão da população.

5 Como exemplo, cinco itens do Teste RA são apresentados no Apêndice.

Sobre os autores 
36 W. B. Andriola e L. R. Cavalcante

ção (FACED) da Universidade Federal do Ceará e coordenador do LABPAM/UF. Endereço para correspondência: UFC/FACED/Departamento de Fundamentos da Educação/Mestrado em Avaliação Educacional, rua Waldery Uchoa, 1, Benfica, Fortaleza, CE, CEP 60020-110. Fone/Fax: (085) 2833926. E-mail: andriola@ufc.br. Luanna Rodrigues Cavalcante é estudante do curso de Graduação em Psicologia da UFC e Bolsista de Iniciação Científica do CNPq. 


\section{Apêndice}

Exemplos de itens do Teste de Raciocínio Abstrato (RA)

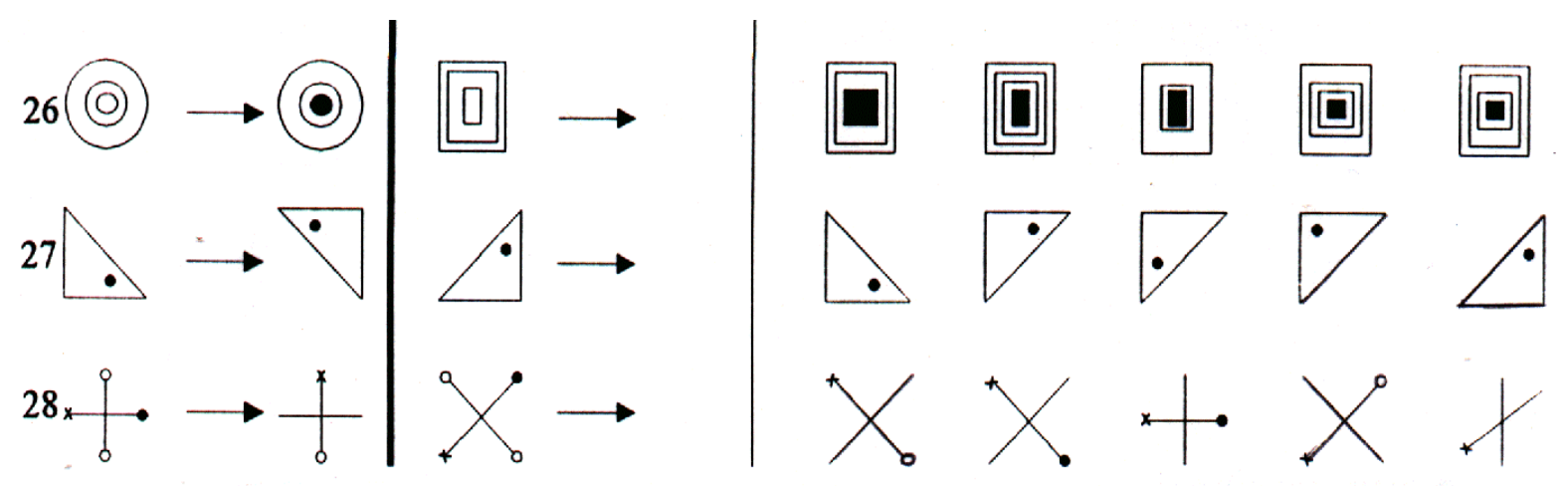

